\title{
e-shape - EUROGEO SHOWCASES: APPLICATION POWERED BY EUROPE CONTRIBUTION TO EUROGEO AND TO THE DEVELOPMENT OF THE EO INDUSTRY
}

\author{
Thierry Ranchin ${ }^{(1)}$, Lionel Ménard ${ }^{(1)}$, Nicolas Fichaux ${ }^{(1)}$, Mathieu Reboul ${ }^{(2)}$ and all the e-shape \\ partners $^{(3)}$
}

${ }^{(1)}$ MINES ParisTech, PSL University / ARMINES, ${ }^{(2)}$ ARMINES, ${ }^{(3)}$ see https://www.eshape.eu/index.php/team

\begin{abstract}
e-shape is a unique initiative under the EU-funded Horizon 2020 programme driven by the need to develop operational Earth Observation (EO) services with and for the users and to create a conducive environment whereby the strengths of Europe are exploited towards addressing societal challenges, fostering entrepreneurship and supporting sustainable development. e-shape is also a flagship project for the European Union (EU) to contribute through worldwide efforts to GEO (Group on Earth Observations) by establishing EuroGEO and by providing its experience and knowledge to the EO community. In this paper, e-shape's objectives and challenges are presented focusing on the development of EO services and promotion European EO capabilities with and for the users.
\end{abstract}

Index Terms - Operational EO services, EuroGEO, codesign, Group on Earth Observations, Earth Observation industry

\section{INTRODUCTION}

Earth Observation (EO) can be defined as all means of measurements and set of information that can be collected about our environment. It includes satellites data and in-situ measurements, encompassing data from the Internet of Things (IOT), citizen science but also all the outputs of the large environmental models (such as meteorological or ocean models) describing our environment. The exploding data sets provided by all this sensors and modeling tools and the means of exploiting them have reached a level of development that allows to envision a future where decisions and actions for the benefit of humankind are informed by coordinated, comprehensive and sustained Earth observations. To achieve this future, there is a need to develop operational, sustainable and users' driven services and to exploit the latest information and technologic tools available.

e-shape is driven by this need. e-shape aims at developing operational EO services with and for the users and to create a conducive environment for addressing societal challenges, fostering entrepreneurship and supporting sustainable development.

e-shape is also a flagship project for the European Union (EU) to contribute through worldwide efforts to GEO (Group on Earth Observations). In this regard, e-shape contributes to establish EuroGEO, the European regional GEO, with the aim to strengthen the benefits of GEOSS (the Global Earth Observation System of Systems developed by GEO) for Europe and contributes to the EuroGEO activities by providing its experience and knowledge to the EO community.

e-shape aims at setting-up and promoting a sustainable organization dedicated to users' uptake of European EO resources, building on Copernicus and GEOSS through the development of pilots supported by co-design methods tailored to Earth Observation (i.e. application-oriented products, services or solutions) built on a user-centric approach and delivering economic, social and policy value to Europe and worldwide.

e-shape is not another $\mathrm{H} 2020$ project. e-shape is an innovation action built as a legacy of previous EU projects and built to make the EO services reaching the operation and the sustainability.

\section{OBJECTIVES}

The e-shape's objectives are fully aligned with the EuroGEO initiative [1, 2, 3] developed by the European Commission in the framework of the Group on Earth Observations (GEO) and strongly contribute to EuroGEO.

e-shape implement a coordinated and comprehensive EO data exploitation initiative through innovation and collaboration amongst the European GEO Members and Participating Organizations in order to accelerate the users' uptake of open EO data and information for the benefit of Europe and the World.

e-shape's general objectives are to set-up and promote a sustainable organization dedicated to users' uptake of European EO resources, building on Copernicus and GEOSS through the development of co-design pilots (i.e. application-oriented products, services or solutions) built on 
a user-centric approach and delivering economic, social and policy value to European citizens.

The main objectives developed in e-shape are to:

- Develop operational EO services with and for users active in key societal sectors. e-shape follow a usercentric approach in which pilots across 7 thematic areas (food security, health, renewable energy, biodiversity, water resources, disaster resilience and climate) aligned with UN sustainable development goals (SDG) [4] will be co-designed from the very beginning of the project [5]. Building on key results from existing GEO actions and on the exploitation of Copernicus Services, e-shape strives to bring operational EO pilots to the market. For each pilot, the teams of scientists, IT developers, user uptake specialists and business experts work closely together with users to design and develop services that can be incorporated in their operational workflows.

- Demonstrate the benefits of the EO pilots through the coordinated downstream exploitation of EO data and the utilization of existing EO resources. The 27 pilots developed within e-shape, demonstrate the benefits for the different users and the iterative improvement of the services provided. To that end, it exploits the IT capabilities and the wealth of data made available through DIAS [6], GEOSS platform [7], NextGEOSS [8], EOSC, in-situ observatories, citizen observatories and any other existing hubs or platforms. This is done in full compliance to the INSPIRE directive, GEO Recommendations on interoperability and GEO Data sharing and Data management principles [9].

- Promote the uptake of pilots at national and international scale, across vertical markets (private and public) and amongst key user communities. eshape places strong emphasis in the uptake of the pilots across three main inter-connected dimensions: Sectorial uptake, National/regional uptake, and International uptake. This is guided by a comprehensive user uptake strategy. It aims at attracting and involving the key organizations (UN-SDGs, GEO, Copernicus, ESA, UNFCCC), initiatives and user communities at large. eshape is also undertaken a series of capacity building activities with the aim to train users in a given sector on the integration of EO-based solutions in their workflow [10].

- Enable the long-term sustainability of the numerous pilots, their penetration in public and private markets and support their upscaling. The sustainable uptake and exploitation of the provided pilots in different markets serving public/government and private users lies at the core of our approach [10]. To boost the sustainability potential of the pilots e-shape supplies all the necessary tools (business plan support, IPR advice, market intelligence, investment readiness, on-line market presence). The penetration of pilots in public and private markets will be further supported by dedicated socio-economic analyses based on an EO value tree helping to raise awareness on the complex benefits of European GEOSS actions to policy makers, public organisations and commercial downstream markets [10].

- Increase uptake by raising awareness on the solutions developed through tailored and welltargeted communication, dissemination and outreach activities [11]. The impact of large projects involving stakeholders with different backgrounds (e.g. EO-savvy vs. non-EO-conversant), thematic expertise (food, energy, environment, etc.), motivation (market success vs. policy implementation) and languages, can be significantly increased if the activities are wellcommunicated through targeted interactions, fit-forpurpose communication practices (e.g. social media, brochures, animations, etc.) and visually powerful media. e-shape undertakes a forward-looking and innovative set of outreach activities including an helpdesk (http://helpdesk.e-shape.eu) raising awareness on all its offers, providing impetus to improved policy making ignited from uptake of Copernicus and GEO in Europe and beyond and making the link between the different stakeholders and services providers.

In addition, e-shape aims at creating strategic knowledge linked with its main objectives and to offer this knowledge to the Earth Observation community and industry to allow the dissemination and communication on good practices, with papers, recommendations, etc.

\section{SHOWCASES AND PILOTS}

The 7 thematic areas of e-shape i.e. food security, health, renewable energy, biodiversity, water resources, disaster resilience and climate are the showcases and support the three main priorities engagement of GEO, i.e. the United Nations 2030 Agenda for Sustainable Development, the Paris Agreement, and the Sendai Framework for Disaster Risk Reduction. To ensure the involvement of the users in eshape, each showcase embedded them in their pilots. 27 initial pilots have been selected ensuring a large sampling of applications, uses-cases, architectures, user communities, level of development, thematic, business models, sustainability challenges, etc. representative of the variety of EO services to public and private sectors. As examples, eshape develops pilots related to:

- Agro-industry by providing services able to support agriculture activities to resist and adapt to climate change and at the same time ensuring the increase of productivity in a sustainable manner

- $\quad$ EO based surveillance of mercury in the framework of the Minamata Convention by developing a transnational 
EO system through the integration of real-time monitoring data from various platforms, modelling tools and advanced global cyber-infrastructure for data sharing and interoperability

- High photovoltaic penetration at urban scale through a GIS-like tool for grid operators, urban planning decision makers, industries, aggregators for solar energy trading, citizen (PV self-consumption) and researchers, providing an urban energy system modelling of distribution grids to plan, monitor and nowcast (i.e. very short term forecast) the spatiotemporal variability of the electric consumption and of the production of fleet of PV rooftop systems.

- $\quad$ Biodiversity conservation by developing a portal within the GEO BON initiative, one of the flagships of GEO, providing access to the EO enabled Essential Biodiversity Variables (EBV) developed by the biodiversity research community.

- Visibility for Scuba Diving by using of EO data in an user focused downstream services that provides near real time (NRT) visibility information for a specific location based on EO data.

- Volcanic eruptions by improving the critical infrastructure protection capability in Europe and helping to reduce its vulnerabilities in the framework of the EU Directive on European critical infrastructures and assessment.

- $\quad$ Finnish forest industry by forecasting harvesting conditions to handle the seasonal variation of wood supply operations and terrain and forest road trafficability. These issues are causing significant production shortfalls and pose a risk in a future with shorter frost periods due to climate change.

In addition, e-shape develops an on-boarding process allowing to include new pilots in the course of the project, to extend the range of applications and services and to confirm the approaches and methodologies developed.

The full list of pilots is accessible at https://www.eshape.eu/index.php/all-pilots showing the huge potential of EO for supporting various public and private sectors with relevant solutions.

\section{CONCLUSION}

e-shape is a major contribution to the EuroGEO initiative but is also a major innovation action supported by the EU to strengthen the EO sector and to develop highly qualified jobs. e-shape is creating a favorable environment in such a way.
This project has received funding from the European Union's Horizon 2020 research and innovation programme under grant agreement No 820852

\section{REFERENCES}

[1] E. Goor, J. Dusart, M. Van Meerloo, G. Ollier, J. Ramboer, I. Freytag and G. Le Bouler, «EuroGEO - The European Component of GEO”, IGARSS 2021, Brussels, Belgium, July 11 16, 2021

[2] About EuroGEO, https://ec.europa.eu/info/research-andinnovation/knowledge-publications-tools-and-data/knowledgecentres-and-data-portals/eurogeo/about-eurogeo_en.

[3] EuroGEO concept paper, 22 November 2017, https://ec.europa.eu/info/sites/info/files/eurogeoss/eurogeoss_conc ept paper-2017.pdf.

[4] United Nations, THE 17 GOALS. Sustainable Development, https://sdgs.un.org/goals

[5] R. Barbier, S. Ben Yahia, P. Le Masson, B. Weil. “Expanding usages of Earth Observation data: a co-design approach to grow an ecosystem of efficient service designers”, IGARSS 2021, Brussels, Belgium, July 11 - 16, 2021

[6] Data and Information Access Services, https://www.copernicus.eu/en/access-data/dias

[7] The GEOSS platform, https://www.geoportal.org//

[8]NextGEOSS Data Hub, https://catalogue.nextgeoss.eu/

[9] M-F, Voidrot, I. Simonis, R. Barbier, P. Le Masson, N. Fichaux, T. Ranchin, "Looking for reproductibility for Earth Observation Applications at the abstract level”, IGARSS 2021, Brussels, Belgium, July 11 - 16, 2021

[10] E. Mamais, F. Piatto, E. Pajot, "Upscaling European earth observation solutions through a comprehensive portfolio of tools the case of e-shape”, IGARSS 2021, Brussels, Belgium, July 11 16, 2021

[11] The e-shape web site, http://www.e-shape.eu

\section{ACKNOWLEDGMENT}

